\title{
A study of papanicolaou stain and cervical acid phosphatase papanicolaou stain in the detection of cervical intra epithelial neoplasm a comparative study
}

\author{
Rajini T ${ }^{1}$, Subhashish Das ${ }^{2, *}$, Seela S.R ${ }^{3}$ \\ ${ }^{1}$ Senior Resident, ${ }^{2}$ Professor, ${ }^{3} \mathrm{HOD} \&$ Professor, ${ }^{2}$ Dept. of Pathology, ${ }^{3}$ Dept. of Obstetrics and Gynaecology, Sri Devaraj Urs \\ Medical College, Tamaka, Kolar, Karnataka, India
}

*Corresponding Author:

Email: daspathology@gmail.com

\begin{abstract}
Introduction: In order to increase the sensitivity of the routine PAP test, a new test was introduced called the CAP-PAP test. In this test, lysosomal enzyme and cervical acid phosphatase (CAP) enzyme are labeled with conventional PAP test which will help in detecting abnormal squamous cells of cervix. This enzyme can be used as a biomarker for cervical dysplasia when CAP positivity is detected inside abnormal squamous cells of cervix and can also be used as a surrogate in detection of CIN.

Aims: To perform CAP-PAP and PAP staining on cervical smears. To compare CAP-PAP results with PAP results and correlate with cervical histopathology reports.

Methods and Materials: 150 females undergoing cervical cancer screening at OBG Department, of our institute were studied. Two cervical smears each were collected from all patients for PAP and CAP-PAP staining. The results were compared and correlated with respective histopathology reports.

Statistical Analysis Used: Chi-square test, Kappa statistics, $\mathrm{P}$ value of $<0.05$.

Results: 75 PAP positive cases out of which 34 were LSIL, 17 were HSIL, 13 were ASCUS, 9 were ASC-H, and 2 were SCC cases. In the remaining 75 PAP negative smears, 38 cases were inflammatory smears and 37 cases were of NLIM smears. Among the PAP positive cases, CAP-PAP results were similar to PAP results in all the LSIL, HSIL, ASCUS, ASC-H and SCC cases. Among the PAP negative cases, CAP-PAP was negative in all the 37 NILM cases, but of the 38 inflammatory cases, CAP-PAP was negative in 30 cases but was positive in 8 cases. CAP-PAP results had good agreement with PAP results and correlated well with histopathology results.

Conclusion: CAP positivity is identified by presence of res granules in abnormal squamous cells which helps in its early detection. In screening of cervical cancer on a large scale. CAP PAP acts as a quick and efficient method. The test promises a great future in health centers as the technicians can be easily trained for identification of abnormal cells. A large trial is requires before widespread use of CAP-PAP staining can be recommended in routine clinical practice.
\end{abstract}

Keywords: Cervical cancer, Cytology, Cervical acid phosphatase, Screening.

\section{Introduction}

Cervical cancer screening using Papanicolaou (PAP) stained smears have been successful in detecting early cervical cancer and thereby help in the management of cervical cancer cases. ${ }^{1}$ In order to further improve the cervical cancer detection rates test several ancillary test have been suggested, ancillary test such as Polymearase chain reaction tests, evaluation of telomearase repeat activity by the Telomeric Repeat Amplification Protocol (TRAP) and Immunocytochemical detection of P16INK4a protein ${ }^{2}$. The study is done to assess the utility of Cervical Acid Phosphatase-Papanicolaou test (CAP-PAP) as a useful ancillary test for visualization of abnormal squamous cells in relation to cervical histopathology examination.

\section{Aims and Objectives}

1. To perform Cervical Acid PhosphatasePapanicolaou (CAP-PAP) and Papanicolaou (PAP) staining on cervical smears.

2. To compare CAP-PAP results with PAP results.

3. To correlate CAP-PAP and PAP results with histopathology reports

\section{Materials and Methods}

The sources of data were patients undergoing cervical cancer screening at Obstetric and Gynecological Department of our institute duringduring January 2016 to December 2017. This was an opportunistic type of screening method where patients presented with complaints. Informed consent was obtained from the patients after which two smears were collected simultaneously for PAP and CAP-PAP staining.

\section{Inclusion criteria}

1. All patients undergoing cervical cancer screening from whom two cervical smears would be collected

\section{Exclusion criteria}

1. Smears showing atypical glandular cells and adenocarcinoma

2. Cases who have undergone radiation changes.

Sample size: Sample size was estimated by using based on the sensitivity and specificity of PAP at $75 \%$ and $100 \%$ with respect to histopathology findings obtained from the study Neha Batra et al. ${ }^{3}$ Considering $10 \%$ nonresponse rate, sample size of $136 \pm 13.6 \approx 150$ cases of Cervical histopathology will be included in the study. ${ }^{4}$ 


\section{Results}

A total of 1596 patients underwent cervical cancer screening at Obstetric and Gynecological Out Patient Department of our institute during Jan 2016 to December 2017. The present study included 75 PAP Positive and 75 PAP Negative patients, a total of 150 patients.

Age distribution of all the subjects in the study is shown in Table 2. Among PAP positive cases, 58.7\% were above 40 years as shown in Table 1 . Among the 150 patients studied, $49.3 \%$ had WDPV, $18 \%$ had Mass PV, 19.3\% underwent routine screening, $6 \%$ had pain abdomen, $6 \%$ had bleeding PV and $1.3 \%$ had burning Micturition. Of those with PAP negative, $52 \%$ had WDPV, $25.3 \%$ had Mass PV, 9.3\% had routine screening, $6.7 \%$ had pain abdomen, $4 \%$ had bleeding PV and $2.7 \%$ had burning Micturition. Among those with PAP positive, $46.7 \%$ had WDPV, $10.7 \%$ had Mass $\mathrm{PV}, 29.3 \%$ had routine screening, 5.3\% had pain abdomen, $8 \%$ had bleeding PV.as shown in Table 2

Distribution of PAP and CAP-PAP results: In our study, there were 75 PAP positive cases out of which 34 were LSIL, 17 were HSIL, 13 were ASCUS, 9 were ASC-H, and 2 were SCC cases. In the remaining 75 PAP negative smears, 38 cases were inflammatory smears and 37 cases were of NLIM smears. Among the PAP positive cases, CAP-PAP result was similar in all LSIL, HSIL, ASCUS ASC-H and SCC cases. Among the PAP negative cases, CAP-PAP was negative in all the 37 NILM cases, but of the 38 inflammatory cases, CAP-PAP was negative in 30 but was positive in 8 cases as shown in Table 3.

Comparison of CAP-PAP and PAP results: The comparative results are shown in Table 4 Statistical analysis showed that CAP PAP had sensitivity of $100 \%$, Specificity of $89.33 \%$, PPV of $90.36 \%$, NPV of $100 \%$ and diagnostic accuracy of $94.67 \%$ as shown in Table 5. As per the Kappa statistic, agreement between CAP PAP and PAP was 0.893 (Almost perfect agreement) which shows that CAP-PAP results were similar to PAP result.

Histopathology Results: Histopathology results showed 71[47.3\%] negative and 79 positive for dysplasia among which 39 [26\%] had CIN1, 18[12\%] had CIN2, 20[13.3\%] had CIN3 and 2[1.3\%] had SCC as shown in Table 6 . The histopathology of various lesions is depicted in Fig. 8.

Comparison between PAP results and Histopathology results: Among 75 subjects who were PAP positive, 74 were positive on histopathology (true positive) but 1 was negative (false positive). Among 75 PAP negative, 70 were negative on histopathology (true negative) but 5 were positive (false negative) as shown in Table 7. The association between PAP and histopathology results was significant but the single false positive case had reactive inflammatory changes and was reported as ASCUS on PAP, while the 5 false negative cases had very few abnormal cells which were unevenly distributed in the smear. Thus, PAP showed lower sensitivity of $93.67 \%$, better specificity of $98.59 \%$, PPV of $98.67 \%$, but lower NPV of $93.3 \%$ and diagnostic accuracy of $96 \%$ as shown in Table 8.

Comparison between CAP PAP results and histopathology results: Among 83 CAP-PAP positive subjects, 79 [94.4\%] were positive on histopathology (True positive) and 4 [5.6\%] negative (false positive). Of the remaining 67 CAP-PAP negative, $100 \%$ were histopathology negative (True negative) and none were positive [no false negative] as shown in Table 9. False positive with CAP-PAP were seen in 3 cases of florid inflammation and one case showed misinterpretation of endocervical as ASCUS.

There was significant association between CAP PAP and histopathology reports with sensitivity of $100 \%$, specificity of $94.37 \%$, PPV of $95.18 \%$, NPV of $100 \%$ and diagnostic accuracy of $97.33 \%$. Agreement between CAP PAP result and histopathology results was 0.946 which was almost perfect agreement as shown in Table 10.

\section{Discussion}

In our study, there were 75 PAP positive smears out of which 34 were LSIL, 17 were HSIL, 13 were ASCUS, 9 were ASC-H, and 2 were SCC cases. In the remaining 75 PAP negative smears, 38 cases were inflammatory smears and 37 cases were of NILM smears.

Comparison of CAP-PAP and PAP Results: Similar to Niranjan et $\mathrm{al}^{4}$ our study also showed considerable agreement of around $97 \%$ between CAP-PAP and PAP with all 75 cases showing CAP-PAP positivity and among those with PAP stain negative $8[10.7 \%]$ were CAP PAP positive and 67 [89.3\%] were CAP PAP negative. Hence, CAP-PAP was positive in 83 cases and negative in 67 cases.

Correlation of PAP results with histopathology: Among 75 subjects who were PAP positive, 74 were positive on histopathology and one was negative. Of the 75 PAP negative subjects, 70 were negative on histopathology but 5 were positive. Our concordance rate was $92 \%$ which is similar to that of Nawaz et al ${ }^{5}$ $(74 \%)$, Yeoh et $\mathrm{al}^{6}(52 \%)$ and Rasbridge et $\mathrm{al}^{7}(81.2 \%)$ respectively. This shows a good correlation between pap smear and histopathology and was compared with other studies Table 11

Correlation of CAP-PAP Results with Histopathology: Among 67 CAP PAP negative cases, all of them were negative on histopathology and among the 83 CAP PAP positive cases, 79 were positive on histopathology but 4 were negative.

There was significant association between CAP PAP and histopathology. CAP PAP had sensitivity of $100 \%$, Specificity of $94.37 \%$, PPV of $95.18 \%$, NPV of $100 \%$ and Diagnostic Accuracy of $97.33 \%$. Agreement between CAP PAP and histopathology was 0.946 
(almost perfect agreement). This is similar to study done by Batra et $\mathrm{al}^{3}$ and Deb et $\mathrm{al}^{8}$ Table 12 .

Our study revealed that CAP-PAP detected 8 cases which PAP could not detect out of which 5 cases were asymptomatic [routine screening] and 3 had symptoms [WDPV].

Table 1: Age distribution of all subjects in the study

\begin{tabular}{|c|c|c|c|}
\hline \multicolumn{1}{|c|}{} & Count & \% \\
\hline \multirow{4}{*}{ Age } & $<30$ years & 30 & $20.0 \%$ \\
\cline { 2 - 4 } & $31-40$ years & 50 & $33.3 \%$ \\
\cline { 2 - 4 } & $41-50$ years & 41 & $27.3 \%$ \\
\cline { 2 - 4 } & $51-60$ years & 12 & $8.0 \%$ \\
\cline { 2 - 4 } & $61-70$ years & 11 & $7.3 \%$ \\
\cline { 2 - 4 } & $>70$ years & 6 & $4.0 \%$ \\
\cline { 2 - 4 } & Total & 150 & $100.0 \%$ \\
\hline
\end{tabular}

Table 2: Comparison of Presentation with respect to PAP results

\begin{tabular}{|c|c|c|c|c|c|}
\hline \multirow{2}{*}{} & \multicolumn{4}{|c|}{ Group } \\
\cline { 3 - 6 } \multicolumn{2}{|c|}{} & \multicolumn{2}{|c|}{ PAP Negative } & \multicolumn{2}{c|}{ PAP Positive } \\
\cline { 3 - 6 } & Count & $\%$ & Count & $\%$ \\
\hline \multirow{3}{*}{$\begin{array}{c}\text { Presenting } \\
\text { complaint }\end{array}$} & Bleeding PV & 3 & $4.0 \%$ & 6 & $8.0 \%$ \\
\cline { 2 - 6 } & Burning Micturition & 2 & $2.7 \%$ & 0 & $0.0 \%$ \\
\cline { 2 - 6 } & Mass PV & 19 & $25.3 \%$ & 8 & $10.7 \%$ \\
\cline { 2 - 6 } & Pain abdomen & 5 & $6.7 \%$ & 4 & $5.3 \%$ \\
\cline { 2 - 6 } & Routine screening & 7 & $9.3 \%$ & 22 & $29.3 \%$ \\
\cline { 2 - 6 } & WDPV & 39 & $52.0 \%$ & 35 & $46.7 \%$ \\
\hline
\end{tabular}

$\chi 2=15.56, \mathrm{df}=5, \mathrm{p}=0.008^{*}$

Table 3: Distribution of PAP and CAP-PAP results

\begin{tabular}{|c|c|c|c|c|}
\hline Group & Lesions & $\begin{array}{c}\text { No of } \\
\text { PAP cases }\end{array}$ & $\begin{array}{c}\text { No of } \\
\text { CAP - PAP } \\
\text { Positive cases }\end{array}$ & $\begin{array}{c}\text { No of } \\
\text { CAP - PAP } \\
\text { Negative cases }\end{array}$ \\
\hline \multirow{3}{*}{$\begin{array}{c}\text { PAP } \\
\text { Positive }-75\end{array}$} & ASCUS & 13 & 13 & 0 \\
\cline { 2 - 5 } & LSIL & 34 & 34 & 0 \\
\cline { 2 - 5 } & ASC-H & 9 & 9 & 0 \\
\cline { 2 - 5 } & HSIL & 17 & 17 & 0 \\
\cline { 2 - 5 } & SCC & 2 & 2 & 37 \\
\hline \multirow{2}{*}{$\begin{array}{c}\text { PAP } \\
\text { Pegative }-75\end{array}$} & Inflammatory & 37 & 0 & 30 \\
\cline { 2 - 5 } & Smears & & 8 & \\
\hline
\end{tabular}

Table 4: Comparison between CAP PAP and PAP results

\begin{tabular}{|c|c|c|c|c|c|}
\hline \multicolumn{2}{|c|}{} & \multicolumn{4}{|c|}{ PAP } \\
\cline { 3 - 6 } & \multicolumn{2}{|c|}{ Positive } & \multicolumn{2}{c|}{ Negative } \\
\cline { 2 - 6 } \multicolumn{2}{|c|}{} & Count & $\%$ & Count & $\%$ \\
\hline CAP- & Positive & 75 & $100.0 \%$ & 8 & $10.7 \%$ \\
\cline { 2 - 6 } PAP & Negative & 0 & $0.0 \%$ & 67 & $89.3 \%$ \\
\hline
\end{tabular}

$\chi 2=121.084, \mathrm{df}=1, \mathrm{p}<0.001^{*}$

Table 5: Validity of CAP PAP results with respect to PAP results

\begin{tabular}{|l|c|c|}
\hline \multicolumn{1}{|c|}{ Parameter } & Estimate & $\begin{array}{c}\text { Lower - Upper 95\% } \\
\text { Cis }\end{array}$ \\
\hline Sensitivity & $100 \%$ & $95.13,100$ \\
\hline Specificity & $89.33 \%$ & $80.34,94.5$ \\
\hline Positive Predictive Value & $90.36 \%$ & $82.12,95.03$ \\
\hline
\end{tabular}




\begin{tabular}{|l|c|c|}
\hline Negative Predictive Value & $100 \%$ & $94.58,100$ \\
\hline Diagnostic Accuracy & $94.67 \%$ & $89.83,97.27$ \\
\hline Cohen's kappa (Unweighted) & 0.8933 & $0.7342-1.052$ \\
\hline
\end{tabular}

Table 6: Histopathology results among study subjects

\begin{tabular}{|c|c|c|c|}
\hline & Count & $\%$ \\
\hline \multirow[t]{5}{*}{ Histopathology Report } & Negative & 71 & $47.3 \%$ \\
\hline & CIN1 & 39 & $26.0 \%$ \\
\hline & CIN2 & 18 & $12.0 \%$ \\
\hline & CIN3 & 20 & $13.3 \%$ \\
\hline & $\mathrm{SCC}$ & 2 & $1.3 \%$ \\
\hline
\end{tabular}

Table 7: Comparison between PAP and histopathology results

\begin{tabular}{|c|c|c|c|c|c|}
\hline \multirow{2}{*}{} & \multicolumn{3}{|c|}{ Histopathology Results } \\
\cline { 3 - 6 } \multicolumn{2}{|c|}{} & \multicolumn{2}{|c|}{ Positive } & \multicolumn{2}{c|}{ Negative } \\
\cline { 3 - 6 } \multicolumn{2}{|c|}{} & Count & $\%$ & Count & $\%$ \\
\hline \multirow{2}{*}{$\begin{array}{c}\text { PAP } \\
\text { Results }\end{array}$} & Positive & 74 & $98.6 \%$ & 1 & $1.4 \%$ \\
\cline { 2 - 6 } & Negative & 5 & $6.7 \%$ & 70 & $93.3 \%$ \\
\hline
\end{tabular}

$$
\chi 2=127.322, \mathrm{df}=1, \mathrm{p}<0.001^{*}
$$

Table 8: Validity of PAP results with respect to Histopathology results

\begin{tabular}{|l|c|c|}
\hline \multicolumn{1}{|c|}{ Parameter } & Estimate & $\begin{array}{c}\text { Lower - Upper } \\
\text { 95\% Cis }\end{array}$ \\
\hline Sensitivity & $93.67 \%$ & $86.02,97.27$ \\
\hline Specificity & $98.59 \%$ & $92.44,99.75$ \\
\hline Positive Predictive Value & $98.67 \%$ & $92.83,99.76$ \\
\hline Negative Predictive Value & $93.33 \%$ & $85.32,97.12$ \\
\hline Diagnostic Accuracy & $96 \%$ & $91.55,98.15$ \\
\hline Cohen's kappa (Unweighted) & 0.92 & $0.7602-1.08$ \\
\hline
\end{tabular}

Table 9: Comparison between CAP PAP results and Histopathology results

\begin{tabular}{|c|c|c|c|c|c|}
\hline \multicolumn{2}{|c|}{} & \multicolumn{3}{|c|}{ Histopathology results } \\
\cline { 3 - 6 } \multicolumn{2}{|c|}{} & \multicolumn{2}{|c|}{ Positive } & \multicolumn{2}{c|}{ Negative } \\
\cline { 3 - 6 } & Count & \% & Count & $\%$ \\
\hline \multirow{2}{*}{$\begin{array}{c}\text { CAP PAP } \\
\text { results }\end{array}$} & Positive & 79 & $94.4 \%$ & 4 & $5.6 \%$ \\
\cline { 2 - 6 } & Negative & 0 & $0.0 \%$ & 67 & $100.0 \%$ \\
\hline
\end{tabular}

$$
\chi 2=134.72, \mathrm{df}=1, \mathrm{p}<0.001^{*}
$$

Table 10: Validity of CAP PAP results with respect to Histopathology results

\begin{tabular}{|l|c|c|}
\hline \multicolumn{1}{|c|}{ Parameter } & Estimate & $\begin{array}{c}\text { Lower - Upper } \\
\mathbf{9 5 \%} \text { Cis }\end{array}$ \\
\hline Sensitivity & $100 \%$ & $95.36,100$ \\
\hline Specificity & $94.37 \%$ & $86.39,97.79$ \\
\hline Positive Predictive Value & $95.18 \%$ & $88.25,98.11$ \\
\hline Negative Predictive Value & $100 \%$ & $94.58,100$ \\
\hline Diagnostic Accuracy & $97.33 \%$ & $93.34,98.96$ \\
\hline Cohen's kappa (Unweighted) & 0.9464 & $0.7866-1.106$ \\
\hline
\end{tabular}

Table 11: Comparison of the PAP statistics with of various studies

\begin{tabular}{|l|c|c|c|c|c|}
\hline \multicolumn{1}{|c|}{ Study } & $\begin{array}{c}\text { Sensitivity } \\
\mathbf{\%}\end{array}$ & $\begin{array}{c}\text { Specificity } \\
\mathbf{\%}\end{array}$ & $\begin{array}{c}\text { PPV } \\
\mathbf{\%}\end{array}$ & $\begin{array}{c}\text { NPV } \\
\mathbf{\%}\end{array}$ & $\begin{array}{c}\text { Diagnostic } \\
\text { Accuracy \% }\end{array}$ \\
\hline Present study & 93.67 & 98.59 & 98.67 & 93.33 & 96 \\
\hline Mallur et al $^{23}$ & 41.66 & 81.2 & 86.21 & 78.26 & 40 \\
\hline Jain et al $^{24}$ & 78 & 26.9 & 91 & 11.3 & 73.2 \\
\hline Ashmita et al $^{25}$ & 19.51 & 83.3 & 80 & 86.5 & 23.26 \\
\hline
\end{tabular}




\begin{tabular}{|c|c|c|c|c|c|}
\hline Chaudary et $\mathrm{al}^{26}$ & 25.4 & 99.27 & 94.12 & 74.3 & 76 \\
\hline Naik et al ${ }^{27}$ & 79.4 & 58.3 & 86.1 & 46.6 & 74.5 \\
\hline Saha et $\mathrm{al}^{28}$ & 76 & 83.3 & 86.4 & 71.4 & 79.1 \\
\hline
\end{tabular}

Table 12: Comparison of the CAP-PAP results of various studies

\begin{tabular}{|l|c|c|c|c|c|}
\hline \multicolumn{1}{|c|}{ Study } & $\begin{array}{c}\text { Sensitivity } \\
\mathbf{\%}\end{array}$ & $\begin{array}{c}\text { Specificity } \\
\mathbf{\%}\end{array}$ & $\begin{array}{c}\text { PPV } \\
\mathbf{\%}\end{array}$ & $\begin{array}{c}\text { NPV } \\
\mathbf{\%}\end{array}$ & $\begin{array}{c}\text { Diagnostic } \\
\text { Accuracy \% }\end{array}$ \\
\hline Present study & 100 & 94.37 & 95.18 & 100 & 97.33 \\
\hline Deb et al & & & & &
\end{tabular}

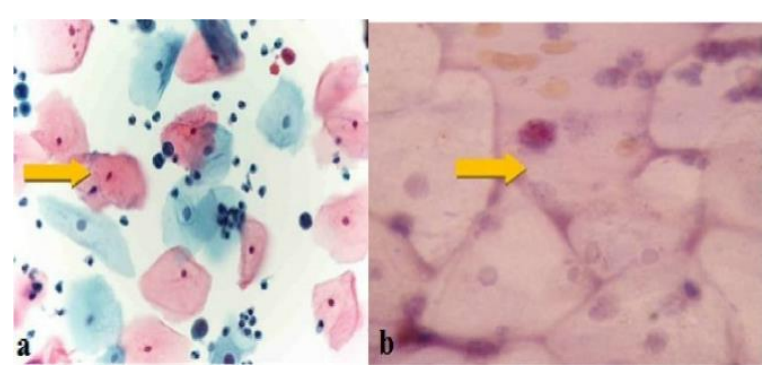

Fig. 1a: PAP Staining of NILM Smear (40X); 1b: CAP-PAP Staining of NILM Smear (40X)

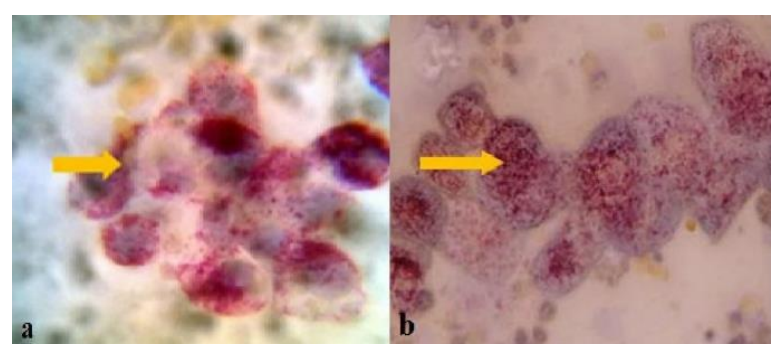

Fig. 2a: Endocervical cells showing CAP positivity which act as an internal control. (CAP PAP, Oil immersion) (100X); 2b: Metaplastic Squamous cells CAP positivity which act as an internal control. CAP stains (40X)

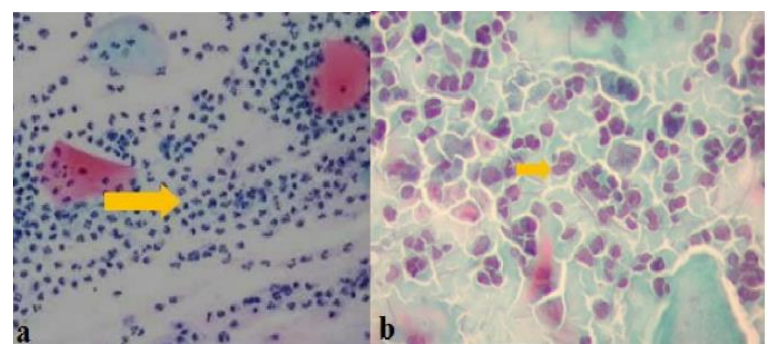

Fig. 3a: PAP staining of inflammatory smear (40X); 3b: CAP-PAP staining of inflammatory smear (100X)

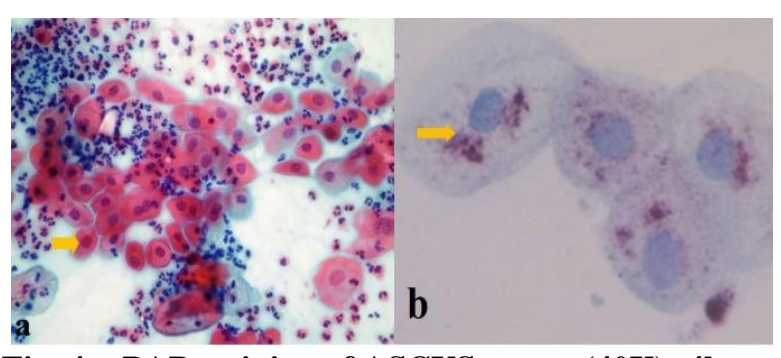

Fig. 4a: PAP staining of ASCUS smear (40X); 4b: CAP-PAP staining of ASCUS smear (100X)

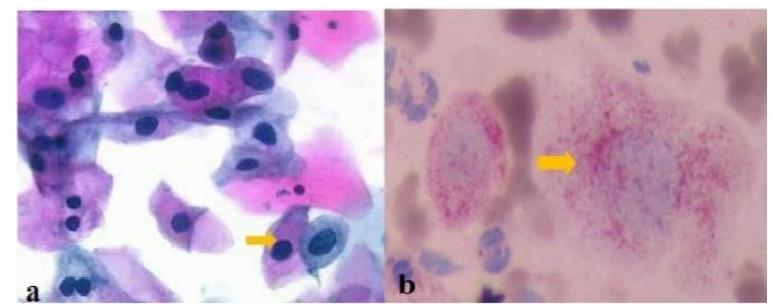

Fig. 5a: PAP staining of LSIL smear (40X); 5b:

CAP-PAP staining of LSIL Smear (100X)

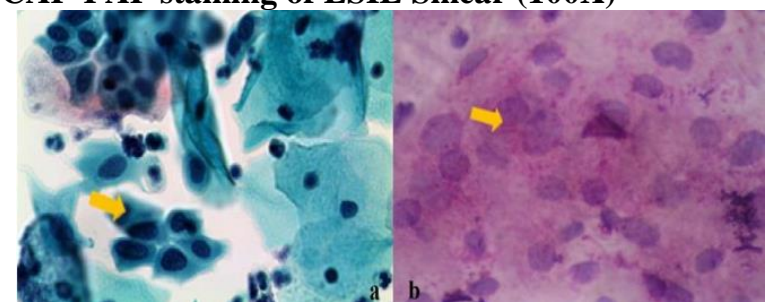

Fig. 6a: PAP Staining of ASC-H Smear (40X); 6b: CAP-PAP staining of ASC-H Smear (100X)

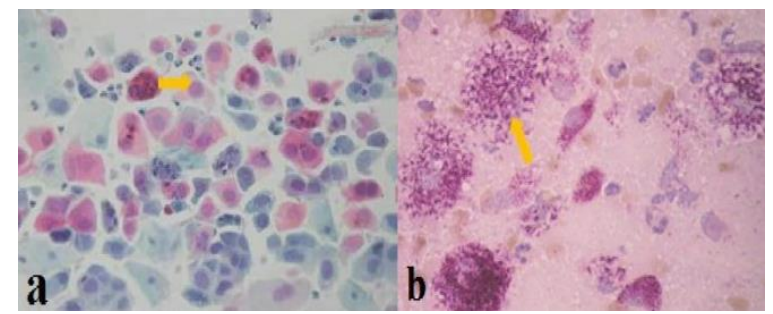

Fig. 7a: PAP staining of HSIL smear (40X); 7b: CAP-PAP staining of HSIL smear (100X) 


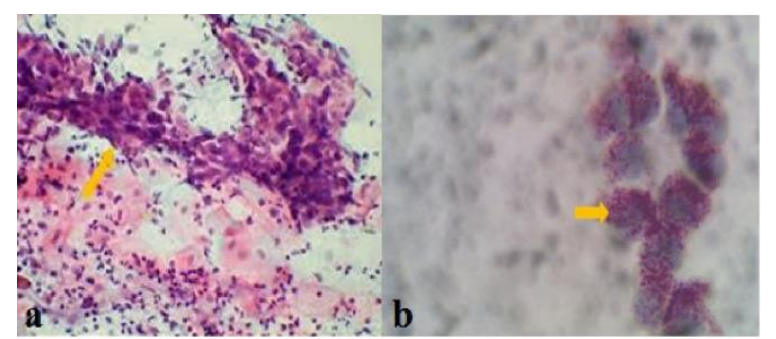

Fig. 8(a): PAP staining of SCC smear (40X); 8(b): CAP-PAP staining of SSC Smear (40X)

\section{Conclusion}

The study was done to assess the role of CAP PAP staining in detection of cervical epithelial abnormalities in comparison with routine PAP staining. The study included both PAP positive and negative patients with histopathology reports as gold standard. It was found that, CAP-PAP had better sensitivity and specificity in detecting cervical epithelial abnormalities when compared to PAP staining. Also, CAP-PAP had better correlation with histopathology reports. Overall CAPPAP staining performance was better in early detection of cervical epithelial abnormalities.

\section{Conflict of Interest: None}

\section{References}

1. WHO/ICO Information Centre on HPV and Cervical Cancer (HPV Information Centre). Human Papillomavirus and Related Cancers in India. Summary Report 2010;(iv).

2. Safaeian M, Solomon D. Cervical Cancer Prevention Cervical Screening: Science in Evolution. Obstet Gynecol Clin N Am 2007;34(4):739- ix.
3. Batra N, Agarwal, Santwani P.M Study of cap pap versus conventional pap in suspicious cervical lesions. Int J Res Med 2015;4(1);102-08.

4. Niranjan J, Prakash CJ, "Cervical Acid Phosphatase: Evaluation as an Adjuvant to Papanicolaou Smear Screening in Cervical Cancer Detection". J Evid based Med Healthc 2015;2 I(6):714-723.

5. Patra P. Sample size in clinical research, the number we need. Int J Med Sci Public Health 2012;1:5-9.

6. Nawaz FHAQ, Aziz AB, Parvez S, Rizvi J. Prevalence of abnormal papanicolaou smears and cyto histological correlation. A study from Aga khan university hospital, Pakistan. Asia Pac J Clin Oncol 2005;1:128-32.

7. Yeoh GP, Chan KW. The accuracy of papanicolaou smear predictions: cytohistological correlation of 283 cases. Hong Kong Med J 1997;3:373-76.

8. Deb P, Iyer VK, Bhatla N, Markovic O, Verma K. Crevical acid phosphatase Detection: A guide to abnormal cell in cytology smear screening for cervical cancer. J Cytol 2008 2014;25(1):1-5.

9. Markovic O, Markovic N. "Cervical acid phosphatase: a biomarker of cervical dysplasia and a potential surrogate endpoint for colposcopy." Dis Markers 2003-2004;19(6):279-86.

10. Mallur PR, Desai BR, Anita D, Geeta D, Bhavana S, Pallav G. et al. Sequential screening with cytology and colposcopy in detection of cervical neoplasia. J South Asian Feder Obst Gynaec 2009;1:45-8.

How to cite the article: Rajini T, Das S, Seela S.R. A study of papanicolaou stain and cervical acid phosphatase papanicolaou stain in the detection of cervical intra epithelial neoplasm a comparative study Arch Cytol Histopathol Res 2018;3(4):195-200. 